\title{
Interactive Assistance for Tour Planning
}

\author{
Yohei Kurata \\ Department of Tourism Science, Tokyo Metropolitan University \\ 1-1 Minami-Osawa, Hachioji, Tokyo 192-0397, Japan \\ ykurata@tmu.ac.jp
}

\begin{abstract}
It is often difficult for individual tourists to make a sightseeing tour plan because they do not have prior knowledge about the destination. Although several systems have been developed for assisting the user's tour planning, these systems lack interactivity, while demanding a lot of data input from the user. In this paper, we introduce a new computer-aided tour planning system, called CT-Planner, which realizes collaborative tour planning. The system provides several tour plans with different characters and asks the user to give feedback. The feedback is utilized by the system for inferring the user's preferences and then revising the tour plans. This cycle is repeated until the user is satisfied with the final plan. Thanks to this cycle the user does not have to register his profiles in advance. In addition, the system allows the user to specify his special requests, which leads to a more satisfying experience of computer-aided tour planning.
\end{abstract}

Keywords: spatial assistance system, tour planning, personalization, user's preference, human-computer interaction, selective travelling salesman problem

\section{Introduction}

Thanks to the popularization of online reservation systems, it has become much easier for people to arrange their transportation and accommodation by themselves. However, it is still difficult for individual tourists to make a sightseeing tour plan, as they often do not have prior knowledge about the destination. Some guidebooks kindly show typical tour plans, but the problem is that attractive plans differ from person to person due to a large variety of tour preferences. Of course, this problem is not so serious when the destination is a small city or village where possible activities are limited. However, if people make a short visit to a large city with various POIs (points of interest), it becomes a very difficult problem for them to find an efficient tour plan (i.e., the plan that gives high satisfaction to the tourist in a short time) from a large possibility of tour plans.

To save individual tourists from such difficulty, several computer systems for supporting the user's tour planning have been developed. Some systems show a list of attractive POIs in the target area, taking the user's tour preference into account (e.g., [1-3]). However, even if people are informed about attractive POIs, it is still difficult 
for them to make a tour plan without the knowledge about the spatial arrangements of these POIs and the transportations between them. Thus, several systems were equipped with the ability to generate personalized tour plans [4-8]. Unfortunately, at the moment, these systems lack interactivity (e.g., we cannot request the system to insert certain POIs into the recommended tour plan), while demanding a lot of data input from the user (Section 2).

In this paper, we introduce a new computer-aided tour planning system, called $C T$ Planner, which overcomes the usability issues in the previous systems. CT-Planner stands for Collaborative Tour Planner (and also City Tour Planner). The key feature of this system is its collaborative planning process, which models the interaction between a tourist and a human tour advisor. First, the system provides several tour plans, which have different characters (nature-oriented, art-oriented, and so on). Shown these example plans, the user gives certain feedback to the system. From this feedback the system infers the user's preference and then revises the tour plans accordingly. By repeating this process, the recommended tour plan eventually agrees with the user's preference. This cyclic process nicely fits the assistance to ordinary tourists, because they usually do not have concrete requests from the very beginning, but rather the requirements are gradually formed during the process of planning [9]. This idea was briefly introduced in our previous work [10] among a number of ideas for future tourist information systems. Among these ideas, this paper focuses on the idea of the cyclic tour planning process. We concretize this idea in comparison with typical interactions between a tourist and a tour advisor, develops a prototype system that implements the idea, and demonstrates that this idea really works.

In the spatial sciences, many researchers studied problems in route planning (e.g., $[11,12]$ ) and following navigation (e.g., $[13,14])$. In contrast, the tour planning problem has attracted less interest, probably because it looks a domain-specific, application-oriented problem. Nevertheless, we believe the importance of this problem, because tourism is an activity that ordinary people have to handle heavy amount of spatial information to make decisions and they often need assistance from somebody. In addition, as an application of today's mobile and ubiquitous technologies, tourist assistance attracts more and more attention. In such a context, what we should pursue is appropriate assistance for the user's decision making, rather than simply providing more and more location-based information. We therefore consider that tour planning assistance will be one of the key topics for future research on spatial information systems.

The remainder of this paper is structured as follows: Section 2 gives a review of previous tour planning systems. Based on this review Section 3 discusses desirable designs of the systems. Section 4 introduces our implementation and its technical detail. Section 5 demonstrates how a user can make a tour plan in our system. Section 6 discusses the remaining issues toward more intelligent tour planning assistance. Finally, Section 7 concludes with a discussion of future problems. 


\section{Previous Tour Planning Systems}

Most previous tour planning systems take similar three-step approaches for generating a tour plan. First, the system profiles the user's tour preference. Second, the system evaluates all POIs in the target area, considering the user's preference. And lastly, the system generates the 'best' tour plan, considering the calculated values of the POIs and given time/origin/goal constraints.

Profiling the user's preference is a problematic process. Early decision-support systems ask the user to set several preference parameters manually, for instance, by sliders (e.g., $[4,15])$. This interface often frustrates the user, since he is forced to evaluate his own preference under given artificial criteria. To make the preference setting interface more user-friendly, Kurata et al. [5] introduced a questionnaire-based approach. In their system, the user is given fifteen questions, each showing two tour purposes and asking the user's preference on them (Fig. 1). Then, from the answers to these fifteen questions, the system calculates quantitative weights to ten tour purposes (Fig. 2) using AHP [16]. The problem of this approach is the time taken to answer fifteen questions [5]. To realize quicker user profiling, some systems ask the user to input his demographic data (age, gender, occupation, etc.) and calculates his preference parameters, assuming that tourists with demographically similar properties have similar tour preference $[1,8]$. However, this approach also has a risk to annoy some users, giving an impression that the system offends their privacy and has a stereotyped view of their preferences. As an alternative approach, P-Tour [6] asks the user to evaluate the POIs by himself, from which the system generates the tour plan. This approach allows the user to specify his request directly and to skip the problematic profiling process, but it is hard work to estimate and input the attractiveness of all POIs in the target area.

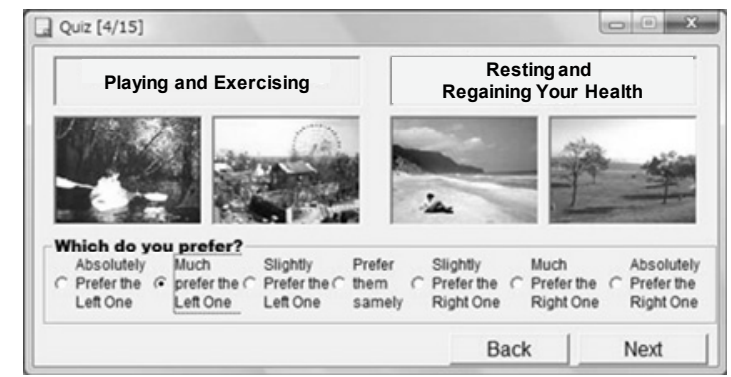

Fig. 1. Questionnaire-based preference profiling in [5]

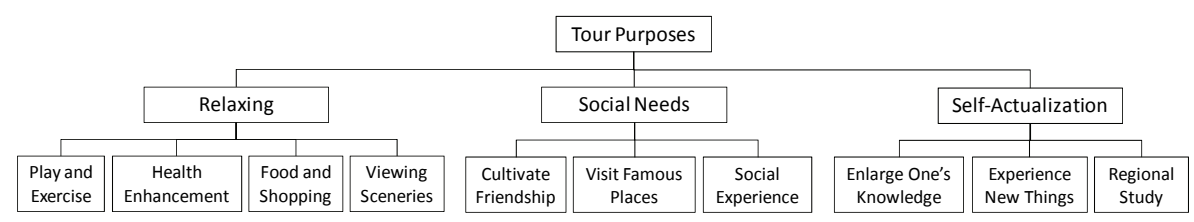

Fig. 2. Structure of ten tour purposes that forms the foundation of the user's preference model in [5] 
Once the user's tour preference has been profiled, the system estimates the value of each POI for the user. The value may be calculated from the matching between the user's preference and the POI's chacter, or from the evaluation of this POI by other tourists with similar properties. In [5], each POI is scored by ten criteria, which correspond to the ten tour purposes in Fig. 2. Thus, by summing the scores of the ten criteria while applying the corresponding weights, the value of each POI for the user is calculated.

Finally, the system computes the 'best' tour plan. In the previous systems, the best tour plan is usually considered to be the plan that maximizes the total values of POIs to be visited during the tour. This problem is represented mathematically as follows (see also the illustration in Fig. 3):

Given a complete graph $(V, E)$, the utility of each node $u_{i}$, the visitation time spent at each node $t^{\text {visit }}{ }_{i}$, the travel time between two nodes $t^{\text {travel }}{ }_{i j}$, origin node $v_{\text {ori }} \in V$, goal node $v_{\text {goal }} \in V$, and time constraint $T$, find a series of nodes to be visited $v_{a_{1}}, \ldots, v_{a_{k}}\left(v_{a_{i}} \in V\right)$ that maximize the sum of utilities $\sum_{i=1}^{k} u_{a_{i}}$ under the following three constraints:

$$
\begin{gathered}
\sum_{i=1}^{k} t^{v i s i t}{ }_{a_{i}}+\sum_{i=0}^{k+1} t^{\text {travel }} a_{i} a_{i+1} \leq T \\
v_{a_{0}}=v_{\text {ori }} \\
v_{a_{k+1}}=v_{\text {goal }}
\end{gathered}
$$

Here each node represents a POI or a transportation node (e.g., a train station). The utility of a POI-node is given as the POI's value for the user, while the utility of a transportation node is set zero. The link and traveling time between each node pair may be computed beforehand in a GIS using the shortest path algorithm. On the other hand, visitation time spent at each POI-node has to be specified manually by experts. From a computational point of view, this problem is essentially the Selective Travelling Salesman Problem (STSP), which is known to be an NP-hard combinatory optimization problem [17]. Thus, the previous systems have adopted approximate methods, such as a genetic algorithm [7], to derive semi-optimal solutions in a practical time.

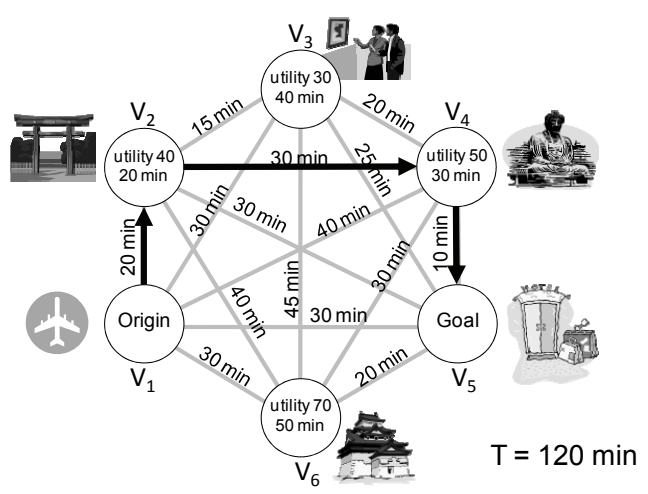

Fig. 3. Illustration of the choice of the 'best' tour plan 


\section{$3 \quad$ Interactive Tour Planning}

Seifert [9] pointed out that the main disadvantage of personalized tour generation is that it excludes the user's participation in the process of planning. Indeed, according to the user test in [5], users complained about the inability to customize the recommended tour plans by adding or removing POIs which they want to visit/avoid. The tour planning systems in $[1,8]$ also have the same problem. Exceptionally, PTour [6] allows the user to express where he wants to visit/avoid by assigning high/low scores to the POIs. However, P-Tour forces the user to spend a lot of time to evaluate all POIs in the target area.

How can we avoid these problems? We can glean some hints from typical interactions between a tourist and a tour advisor. For instance, let us imagine the following conversation:

"I'd like to look around Yokohama in three hours or so."

"Three hours. Okay, then, this is the most popular plan."

"Well, hmm, looks like lots of museums in it. Honestly, I don't have much interest in museums..."

"I see. Then, how about this plan? This visits more scenic sites along the port. Or, how about this plan visiting Sankei-en Park. You can enjoy lots of cherry trees in full bloom right now."

"Hmm. For me, visiting portside scenic points sounds better. Well, but, I'd also like to visit the Port Museum. It's very famous, isn't it?"

"Yes, I definitely recommend it. So, how about this plan? First, you go ..."

Usually a tour advisor does not know the interest of a tourist at the beginning of their conversation; the advisor learns the tourist's interest from the conversation. The tourist's response to recommendations indicates something about his preference. In many cases, the tourist is also not well aware of his interest at the beginning. He develops his interest while viewing the actual plans recommended by the advisor. Thus, experience advisor introduces several plans in a smart order, so that the tourist becomes aware of his own interest and able to judge what plan fits him.

Our system imitates this process; that is, we adopt the cycle where the system presents a set of plans to the user, infers the user's preference from the user's response, and then generates a new set of plans. This cycle for computer-assisted planning is called the candidate/critique model [18]. Although this cycle takes a bit of time to reach a final plan, the user probably attains a high level of satisfaction with the plan, as he can experience that his involvement directly results in the improvement of the plan. Probably the user would not attain such satisfaction, even if the smart system could derive the truly best solution in a single step. Another merit of this cycle is that the user does not have to specify his preference in advance. The user can discover his preference and requests through the comparison of actual plans.

Seifert [9] discussed a similar cyclic model of computer-aided tour planning. The difference from her approach is that our system intentionally generates several plans with different characters in order to seek the user's tour preference, while her system simply generates the solutions - possibly many — that satisfy given constraints. 
In the previous conversation, the tourist said that he did not have an interest in museums, while he requested to visit the Port Museum. Like this example, a tourist sometimes wants to visit a POI of the sort that he usually avoids. Conversely, he sometimes wants to skip a POI of the sort that he/she usually visits. For instance, a tourist, who usually likes modern art but not historical monuments, may request to visit the Arc de Triomphe because it is world-famous, or not to visit Musée d'Orsay because he has been there before. Computer-aided tour planning systems, therefore, should be able to support such case-by-case requests, just like a human tour advisor can do it.

\section{CT-Planner}

Based on the design concepts discussed in Section 3, we developed a new computeraided tour planning system, called CT-Planner (Collaborative Tour Planner). As this name indicates, the system aims at the assistance of user-driven tour planning, rather than computer-based tour optimization. Currently the system is a stand-alone application for Windows PC, but we are also planning its server-client version that can be accessed from mobile devices.

Fig. 4 shows the main screen of CT-Planner. It shows two tour plans. The plan on the left is a recommended plan, which is computed based on the current model of the user's tour preference. The plan on the right is one of alternative plans. We have six alternative plans, namely 30min-shorter plan, 30min-longer plan, more-education plan, more-art plan, more-nature plan, and more-experience plan. The user can browse different alternatives by clicking tabs above the map and, if he wants, he can select the alternative plan that looks more attractive than the recommended plan shown on the left. Based on this selection, the time constraint or the model of the user's preference is updated and accordingly, all plans, including alternative plans, are revised under the new conditions. From the user's viewpoint, it looks that the alternative plan, if it is selected, moves to the left and becomes the next recommended plan. Note that we designed to display only two tour plans each time, following the key idea of AHP [16] that paired comparison is much easier than selecting one preferable plan from a number of alternatives.

If the user clicks the name tag of a node, its properties are shown in a new window (Fig. 5). In this window, the user can specify his request with regard to this node; that is, whether he wants to set this node as the origin/goal of the tour or, if it is a POInode, whether he wants to visit/avoid this POI. The request yields the revision of all tour plans, including alternative plans. Once the user specifies a request, the system accommodates this request until he cancels it, no matter if he selects another alternative plan or changes the tour conditions afterwards.

Through repeated selection of preferable tour plans and/or specification of requests, the recommended plan will eventually become the plan that follows the user's preference and requests. Thus, when the user agrees with the recommended plan, the system prints out the plan and ends the process. 
Our setting is similar to the setting of multi-criteria decision analysis (MCDA) [19], where the user is asked to select the best plan from multiple alternative. MCDA provides several techniques, such as AHP [16], to assign quantitative weights on given criteria. In our setting, education, art, nature, experience, and popularity correspond to the criteria in MCDA. Thus, we can alternatively apply MCDA techniques to decide the weights on five criteria. Actually, our previous system adopted AHP to calculate such weights [5]. We, however, did not apply AHP in the current CT-Planner, because under AHP the user has to compare the criteria themselves, which can be a burden for the user. Instead, we proposed a new technique to estimate the weights on the criteria indirectly through a cyclic process, in which the user can concentrate the comparison of actual plans.

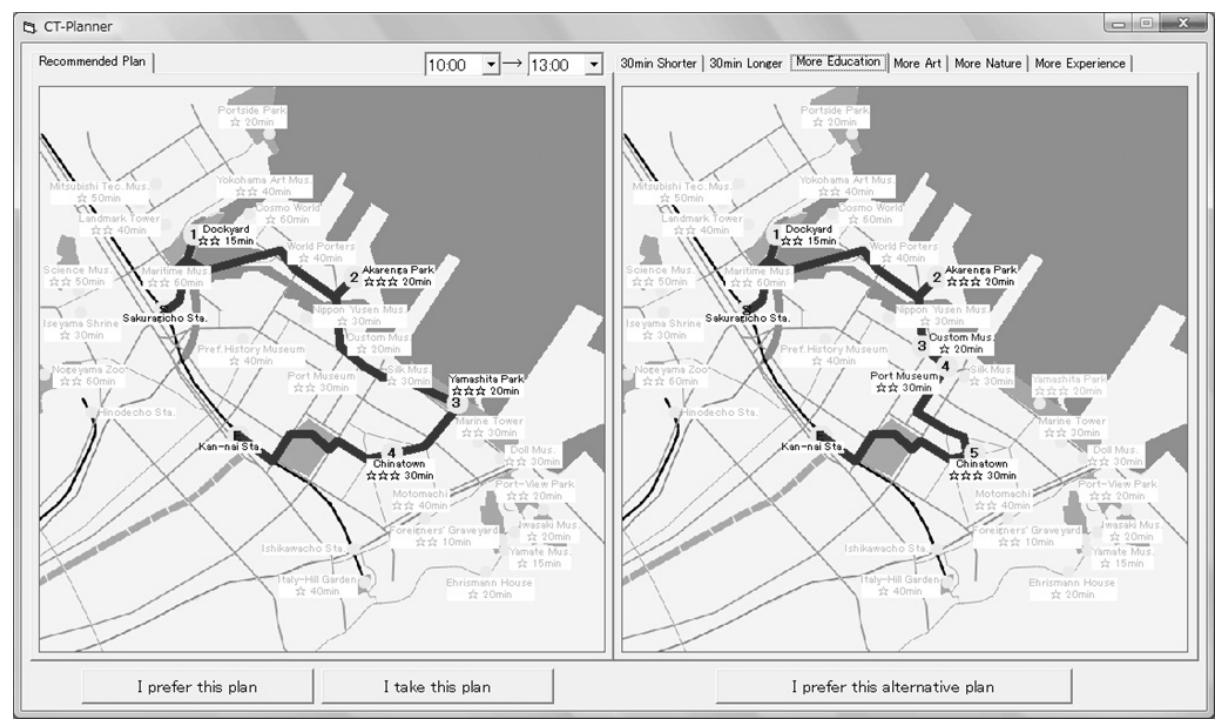

Fig. 4. The main screen of CT-Planner, which shows a recommended plan on the left and one of six alternative plans on the right

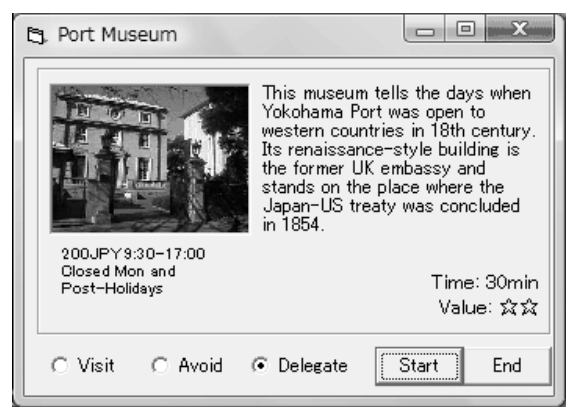

Fig. 5. A window showing the properties of a POI-node, in which the user can specify his request about this node 


\subsection{User's Preference Model}

In CT-Planner, the user's tour preference is modeled by a five-dimensional unit vector $\left(w_{\text {edu }}, w_{\text {art }}, w_{\text {nat }}, w_{\text {exp }}, w_{\text {pop }}\right)$, called the preference vector. Items $w_{\text {edu }}, w_{\text {art }}, w_{\text {nat }}$, and $w_{\text {exp }}$ represent the user's weights on four types of tourist attractions-education, art, nature, and experience. This idea is similar to that of a conceptual vector space [20], in which instances of a concept are represented as points in a vector space. Raubal [20], for instance, represents building façades in a 7D space and considers the distance between instances to see the distinctiveness of each façade from others. Similarly, it is possible in our framework to evaluate the distinctiveness and similarity of tourists using our 5D conceptual vector space.

Initially, the weight is assigned only to popularity; i.e., ( $w_{\text {edu }}, w_{\text {art }}, w_{\text {nat }}, w_{\text {exp }}, w_{\text {pop }}$ ) $=(0,0,0,0,1)$. Naturally, the initial plan on the left becomes a 'standard' plan, which visits popular POIs as much as possible. If the user selects more-education plan, more-art plan, more-nature plan, or more-experience plan, the preference vector rotates toward the corresponding axis by $\theta$ degrees (Fig. 6). Such rotation is repeated if the user selects these plans again. In our current prototype, the parameter $\theta$ is initially assigned 45 degrees. If the user selects the recommended plan shown on the left, $\theta$ is reduced, because this selection means that all alternative plans are too biased for the user. Finally, if the user selects 30min-shorter plan or 30min-longer plan, the preference vector does not rotate.

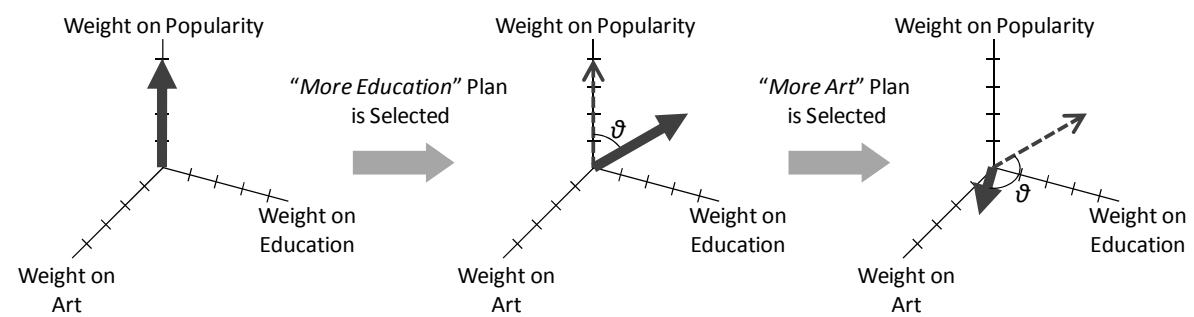

Fig. 6. Adaptation of user's preference model (here only three of five criteria are shown)

The reason why we adopted the user's weights on four types of tourist attractions, $w_{\text {edu }}, w_{\text {art }}, w_{\text {nat }}$, and $w_{\text {exp }}$, for representing the user's preference is that people often express their tour preference in terms of the type of tourist attractions they like. In this paper, we considered that education, art, nature, and experience are the four representative types of tourist attractions, which are highly independent from each other. There are other possible classifications - say, nature, culture, and complex, as used in the classification of UNESCO's world heritage sites, or leisure and sightseeing as used in many travel guidebooks. However, we should avoid detailed classification (say, more than five types), because under such classification we have to provide many tour plans for the user in each cycle, which would overwhelm the user (Section 4.3). Note that we further added one item $w_{\text {pop }}$, which represents the weight on 'popularity', because people typically prefer to visit popular POIs when they do not have any specific request. 


\subsection{Evaluation of POIs}

Each POI has five-grade scores in five criteria-satisfaction level of education, satisfaction level of art, satisfaction level of nature, satisfaction level of experience, and popularity - which are determined by experts in advance. In other words, each POI is represented in another 5D conceptual vector space [20].

The value of the POI for each user is calculated from the total of these scores with the user's weight applied on the corresponding element. This value is calculated every time the user's preference model is updated.

On the screen (Fig. 4), the value of each POI is shown with one to three stars, instead of actual scores, such that the user easily figures out whether the POI is interesting to him or not.

\subsection{Generation of Plans}

For the generation of the recommended tour plan, we adopted the approximate algorithm by Kurata et al. [5]. This algorithm works in $\mathrm{O}\left(n t^{2}\right)$, where $n$ is the number of POIs in the target area and $t$ is the length of tour time. With this algorithm, an ordinary PC generates a semi-optimal tour plan almost instantly. We believe that the optimality of the solution is not a serious problem in our application, since the plan is sufficient enough if it serves a nice draft, which is eventually customized by the user to satisfy his request in detail.

The six alternative plans are calculated by the same process but under slightly different conditions; that is, the 30min-longer plan and 30min-shorter plans are the semi-optimal plans under the 30 minute longer/shorter time constraint, respectively, while the more-education plan, more-art plan, more-nature plan, and moreexperience plans are the semi-optimal plans under the preference vector tentatively rotated toward the corresponding axis by $\theta$ degrees. As a consequence, if the user selects the alternative plan shown on the right, then this plan comes to the left side in the next step.

\section{Demonstration}

Let us observe how CT-Planner works in actual tour planning. Imagine a user who wants to plan a tour in Yokohama (a portside city near Tokyo) starting from Sakuragicho Station at 10:00 and arriving at Kan-nai Station before 13:00. Once the user inputs these basic conditions, the system generates two plans (Fig. 4). As stated before, the left plan is a popularity-based 'standard' plan, while the right plan is one of six alterative plans - for instance, more-education plan. The left plan is generated based on the preference vector $\left(w_{\text {edu }}, w_{\text {art }}, w_{\text {nat }}, w_{\text {exp }}, w_{\text {pop }}\right)=(0,0,0,0,1)$, while the right one is based on $(.707,0,0,0, .707)$. In order to clarify the difference of these two plans, the POIs that only the alternative plan visits (in this case, Custom Museum and Port Museum) are given red name tags on the actual screen, while the POIs that the alternative plan misses (in this case, Yamashita Park) are given green name tags. 
Imagine that the user selects the more-education plan, because he likes museums. Now the selected plan is transferred to the left side, while on the right side the system shows the second generation of more-education plan (Fig. 7a), which is generated based on the preference vector $(1,0,0,0,0)$. He feels that this new plan contains too many museums and thus, he starts browsing other alternative plans. Then, he gets interested in more-experience plan (Fig. 7b) and selects it. Note that this plan emphasizes both education and experience, since the user has selected moreeducation plan in the previous step and accordingly, this plan is generated based on the preference vector $(.5,0,0, .707, .5)$.

Now he notices that the current plan misses Port Museum where he wants to visit. Thus, he clicks the name tag of the Port Museum to open its property window (Fig. 5) and clicks the Visit button. Now the system shows a new plan that visits this museum (Fig. 7c). However, due to the shortage of time, the new plan now skips Akarenga Park, which also looks attractive for him. Thus, he changes the arrival time from 13:00 to 13:30. As a consequence, the plan is revised, such that it contains both the Akarenga Park and the Port Museum (Fig. 7d). Since this plan look satisfactory for him, he clicks "I take this tour" button to print out it and finish planning.

In this way, CT-planner allows the user to make a tour plan in an interactive manner. Compared with the previous computer-aided tour planning systems, the user has much freedom to customize the recommended plans by himself. In addition, the user does not have to set preference parameters or input his demographic data for user profiling.

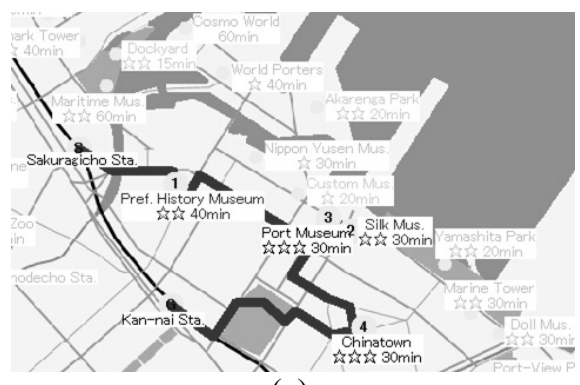

(a)

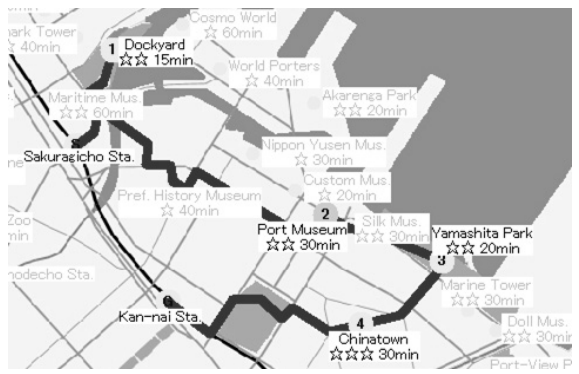

(c)

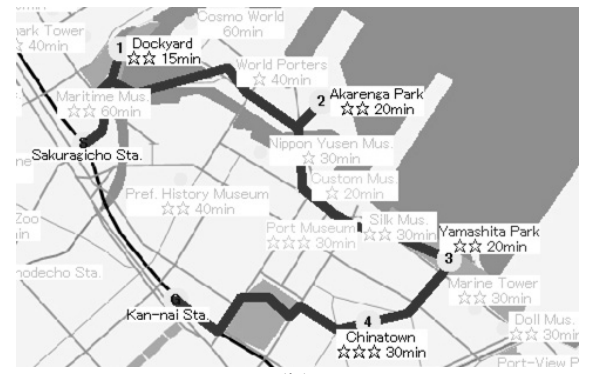

(b)

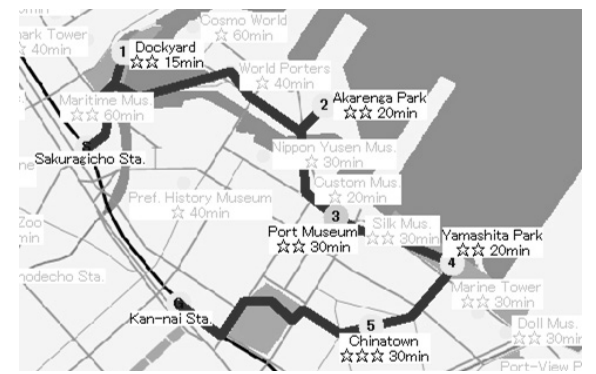

(d)

Fig. 7. Evolution of a tour plan in the scenario of Section 5 


\section{Toward More Intelligent Tour Planning Assistance}

Although CT-Planner realizes easy and flexible tour planning, it has several remaining problems:

1. The attractiveness of routes is not considered.

2. Temporal/seasonal change of POI values is not supported.

3. Travel time between POIs is fixed, even though it may be affected by traffic jams, infrequent transportation service, or the user's choice of transportation modes.

4. Similarly, expected visitation time spent at each POI is fixed regardless of users.

5. Tour plans tend to become monotonous.

The followings are potential solutions to these problems, which revise the ideas discussed in our earlier paper [10].

An intuitive solution for Problem 1 is to assign values not only to nodes, but also to links. With this extension, the problem becomes the Enhanced Profitable Tour Problem (EPTP) [21]. The algorithm for deriving semi-optimal solutions of this problem is already proposed in [21].

Problem 2 is critical for actual tour planning. In the conversation in Section 3, for instance, the tour advisor told that the Sankei-en Park was nice to visit because its cherry trees were in full bloom. There are some POIs, like parks and botanical gardens, whose value changes by seasons. Similarly, there are some POIs, like scenic overlooks, whose value depends on the time of a day. An extreme example is museums, whose values are totally lost when they are closed at night. Thus, it is desirable that the system can support such temporal fluctuation of POI values. Matsuda et al. [22] already tackled Problem 2 (as well as Problem 3) from a computational point of view, introducing time-variant POI values and Fuzzified travel time between nodes.

As for Problem 4, a tourist probably spends more time at the POIs that fit his interest. Also, some people (perhaps older people) prefer slower tours. Thus, it is desirable that the system can estimate the time spent at each POI by each user, for instance, based on a certain statistical model.

Problem 5 arises from the fact that the current system evaluates POIs individually, but not in combinations. For instance, once the system learns that the user is interested in education, the system tends to recommend a tour plan that visits museums for the entire day - which may be boring even for museum lovers. Thus, it will be nice if the system can evaluate the monotony of each tour plan and utilize it for generating tour plans.

\section{$7 \quad$ Conclusions and Future Work}

For the people who travel unfamiliar places, tourist information is vital for their decision making. However, an overwhelming amount of information makes their decision rather difficult. Thus, how to customize the information for individual users is an important issue for tourist assistance systems, which should serve a wide variety 
of users. As an example of such systems, we introduce CT-Planner, with which people can make a tour plan easily as if they are assisted by a human advisor in a tourist information office. A nice feature of computer-aided tour planning systems, in comparison with human advisors, is that the user can consult the systems at any time, as much as he wants, using their own language even in foreign countries.

Our system is considered an example of recommender systems, which have been studied extensively in AI communities and is now used in many online shopping sites [23]. A major technique used in recommender systems is collaborative filtering (CF) [24], in which the system records each user's history of purchase or evaluation of items, finds similar users who have similar histories, and recommends an item that similar user has bought or given high evaluation. CF is particularly useful for the domains where it is difficult to provide explicit features of items and accordingly, it is difficult to model the user's preference (e.g., music and literature). Another merit is that each user does not have to report his/her preference to the system in an explicit manner. Meanwhile, in order to manage a practical CF-based service, we need a huge amount of past user data [23]. Unfortunately, our tour planning domain looks different from other domains where CF works well: First, the tourist's choice of a POI does not mean that he/she likes this POI. For instance, a tourist who has no interest in history may visit a historical site, in case this site is the only POI located between two POIs where he wants to visit. This implies a risk to consider that two tourists who have visited similar POIs have similar preferences. Second, even the same tourist may have different preferences at different times, depending on the destination, motivation, and companions, as found in the user survey of our previous system [5]. For instance, a tourist may prefer art-oriented tourism when she visits Paris by herself, while she may prefer nature-oriented tourism when she visits Switzerland with her husband. Thus, it is risky to rely on the tourist's preference model derived from his/her past behavior. For these reasons, we avoided a CF-based approach and adopted an approach using an explicit model of user's preference. Moreover, considering the instability of tourist's preferences, we decided not to store the profile of each user in the system.

We are planning a systematic user test in near future, which is necessary to substantiate our claim that CT-Planner gives more satisfying tour planning experience than the previous tour planning systems or guidebook-based planning. In this test, we also want to look at psychological aspects of tour planning processes in our user test, hopefully in collaboration with other researchers. For instance, we want to observe each user's interaction with the system - say, how many times he repeats the selection of alternatives and how much he customizes the recommended plan by himself-and seek the patterns of users' decision making. In order to conduct such survey, we are now equipping CT-Planner with the capability of recording the user's operations.

We are also planning to extend CT-planner, such that it works on mobile devices. There a key question is how to provide the service tailored to those devices. For instance, the mobile version of P-Tour [25] monitors the user's location and warns the user if he is off the route or behind schedule. Another idea is to modify the tour plan in case of sudden rain or delay. Such schedule monitoring and real-time rescheduling functions are potential strengths of mobile tour planning systems. In addition, mobile 
tour planning systems will become further useful if they are combined with other intelligent technologies for assisting tourists, such as route-specific route instructions [26] and location-based information querying by pointing [27].

Another potential strength of mobile tour planning systems is availability of trajectory data. Where a user visits and how much time he spends there should tell something about his preference [28]. Of course, a long stay in a certain POI does not necessarily indicate his interest in that kind of POI, because he may actually takes a rest at a café in the POI, for instance [2]. Thus, it is better to use microscopic trajectory data (hopefully both indoor and outdoor data) to analyze the user's action in a POI and seek his interest. The idea of such trajectory-based preference detection is also applicable to other spatial assistance systems. For instance, bike navigation systems may learn from the trajectory what kind of routes the user prefers. In shopping centers, data about where and how long the user spends time may be useful for adapting advertisements to the user. We therefore consider that trajectory-based preference detection is an important topic for the research on spatial assistance systems.

\section{Acknowledgments}

This work was supported by Tokyo Metropolitan University's research fund. We appreciate anonymous reviewers, participants of AGILE'09 Workshop on "Adaptation in Spatial Communication", and participants of GISA-Japan research conference 2009 for their precious comments and encouragement on our work.

\section{References}

1. Ricci, F., Arslan, B., Mirzadeh, N., Venturini, A.: ITR: A Case-Based Travel Advisory System. In: Craw, S., Preece, A. (eds.): ECCBR 2002, Lecture Notes in Computer Science, vol. 2416, pp. 613-627. Springer, Berlin/Heidelberg, Germany (2002)

2. Schmidt-Belz, B., Nick, A., Poslad, S., Zipf, A.: Personalized and Location-Based Mobile Tourism Services. In: Workshop on Mobile Tourism Support Systems, pp. 18-20 (2002)

3. Ardissono, L., Goy, A., Petrone, G., Segnan, M., Torasso, P.: Intrigue: Personalized Recommendation of Tourist Attractions for Desktop and Handset Devices. Applied Artificial Intelligence: Special Issue on Artificial Intelligence for Cultural Heritage and Digital Libraries 17, 687-714 (2003)

4. Kishimoto, H., Mizuno, S.: Development of Tour Planning Support System Using MDL and Genetic Algorithm. In: AI symposium '97, SIG-KBS (in Japanese), pp. 71-76 (1997)

5. Kurata, Y., Okunuki, K., Sadahiro, Y.: Development of a Preference-Based Tour Planning System. In: Papers and Proceedings of the Geographic Information Systems Association (in Japanese), vol. 9, pp. 199-202 (2000)

6. Maruyama, A., Shibata, N., Murata, Y., Yasumoto, K., Ito, M.: A Personal Tourism Navigation System to Support Traveling Multiple Destinations with Time Restrictions. In: IEEE International Conference on Advanced Information Networking and Applications, vol. 2, pp. 18-22 (2004) 
7. Goy, A., Magro, D.: Star: A Smart Tourist Agenda Recommender. In: Configuration Workshop on ECAI 2004, pp. 8/1-8/7 (2004)

8. Lee, J., Kang, E., Park, G.: Design and Implementation of a Tour Planning System for Telematics Users. In: Gervasi, O., Gavrilova, M. (eds.): ICCSA 2007, Lecture Notes in Computer Science, vol. 4707, pp. 179-189. Springer, Berlin/Heidelberg, Germany (2007)

9. Seifert, I.: Collaborative Assistance with Spatio-Temporal Planning Problems. In: Barkowsky, T., Knauff, M., Ligozat, G., Montello, D. (eds.): Spatial Cognition 2006, Lecture Notes in Artificial Intelligence, vol. 4387, pp. 90-106. Springer (2008)

10.Kurata, Y.: Challenges in User-Adaptive Tour Planning Systems. In: Tomko, M., Richter, K.-F. (eds.): AGILE 2009 Workshop on Adaptation in Spatial Communication, SFB/TR 8 Spatial Cognition report series, pp. 19-26 (2009)

11.Mark, D.: Finding Simple Routes: 'Ease of Description' as an Objective Function in Automated Route Selection In: Second Symposium on Artificial Intelligence Applications, pp. 577-581. IEEE Press (1985)

12.Duckham, M., Kulik, L.: "Simplest" Paths: Automated Route Selection for Navigation. In: Kuhn, W., Worboys, M., Timpf, S. (eds.): COSIT '03, Lecture Notes in Computer Science vol. 2825, pp. 169-185. Springer, Berlin/Heidelberg, Germany (2003)

13.Denis, M.: The Description of Routes: A Cognitive Approach to the Production of Spatial Discourse. Current Psychology of Cognition 16, 409-458 (1997)

14.Klippel, A., Tappe, H., Kulik, L., Lee, P.: Wayfinding Choremes: A Language for Modeling Conceptual Route Knowledge. Journal of Visual Languages and Computing 16, 311-329 (2005)

15.Hochmair, H., Rinner, C.: Investigating the Need for Eliminatory Constraints in the User Interface of Bicycle Route Planners. In: Cohn, A., Mark, D. (eds.): COSIT '05, Lecture Notes in Computer Science vol. 3693, pp. 49-66. Springer, Berlin/Heidelberg, Germany (2005)

16.Saaty, T.: The Analytic Hierarchy Process: Planning, Priority Setting, Resource Allocation. McGraw-Hill, New York, USA (1980)

17.Laporte, G., Martello, S.: The Selective Travelling Salesman Problem. Discrete Applied Mathematics 26, 193-207 (1990)

18.Linden, G., Hanks, S., Lesh, N.: Interactive Assessment of User Preference Models: The Automated Travel Assistant. In: Jameson, A., Paris, C., Tasso, C. (eds.): Sixth International Conference on User Modeling, pp. 67-78. Springer, Berlin/Heidelberg, Germany (1997)

19.Malczewski, J.: GIS and Multicriteria Decision Analysis. Wiley (1999)

20.Raubal, M.: Formalizing Conceptual Spaces. In: Varzi, A., Vieu, L. (eds.): FOIS 2004, vol. 114, pp. 153-164. IOS Press, Amsterdam, the Netherlands (2004)

21.Joset, M., Stille, W.: A User-Aware Tour Proposal Framework Using a Hybrid Optimization Approach. In: Voisard, A., Chen, S. (eds.): 10th ACM International Symposium on Advances in Geographic Information Systems, pp. 81-87. ACM Press (2002)

22.Matsuda, Y., Nakamura, M., Kang, D., Miyagi, H.: An Optimal Routing Problem for Sightseeing with Fuzzy Time-Varying Weights. In: IEEE International Conference on Systems, Man and Cybernetics, vol. 4, pp. 3665-3669 (2004)

23.Bachrach, Y., Porat, E., Rosenschein, J.: Sketching Techinique for Collaborative Filtering. In: International Joint Conference on Artificial Intelligence, pp. 2016-2021. AAAI Press (2009)

24.Rensnick, P., Iacovou, N., Suchak, M., Bergstorm, P., Riedl, J.: Grouplens: An Open Architecture for Collaborative Filtering of Netnews. In: ACM 1994 Conference on Computer Supported Cooperative Work, pp. 175-186. ACM Press (1994) 
25.Shiraishi, T., Nagata, M., Shibata, N., Murata, Y., Yasumoto, K., Ito, M.: A Personal Navigation System with Functions to Compose Tour Schedules Based on Multiple Conflicting Criteria. IPSJ Digital Courier 1, 528-536 (2005)

26.Richter, K.-F., Klippel, A., Freksa, C.: Shortest, Fastest, - but What Next? A Different Approach to Route Directions. In: Raubal, M., Sliwinski, A., Kuhn, W. (eds.): Münsteraner GI-Tagen 2004, pp. 205-217. IFGI prints, Münster, Germany (2004)

27.Frank, C.: An Egocentric Spatial Data Model for Intelligent Mobile Geographic Information Systems. Department of Spatial Information Science and Engineering, Master's Thesis. University of Maine, Orono, Maine, USA (2003)

28.Kanda, T., Shiomi, M., Perrin, L., Nomura, T., Ishiguro, H., Hagita, N.: Analysis of People Trajectories with Ubiquitous Sensors in a Science Museum. In: IEEE International Conference on Robotics and Automation, pp. 4846-4853 (2007) 